\title{
Electroreduction of Methyl Cinnamate Using a Rotating Cylinder Electrode. Effect of Mass Transfer on Product-and Stereo-Selectivities
}

\author{
Po-Chung CHENG and Tsutomu NONAKA*
}

Received April 10, 1992 ; Accepted July 1, 1992

\begin{abstract}
Electroreduction of methyl cinnamate (1) in methanol-DMF was carried out galvanostatically with a rotating cylinder $\mathrm{Cu}$ cathode, producing methyl hydrocinnamate (2), dimethyl 3,4-diphenyladipate (3; $d l$ and meso) and methyl trans-2,3-diphenyl-5oxocyclopentanecarboxylate (4), which is stereochemically equivalent to $d l-3$. The ratios of $a l$-dimers $(d l-3$ and trans -4$)$ to meso-3 were found to be ca. 5 , being independent of current density, rotating speed and the amount of acetic acid added. On the other hand, the current density dependence of current efficiency for hydrodimeric products, 3 and $\mathbf{4}$, showed a maximum to which the corresponding current density was related to the rotating speed. On the basis of these results, the role of mass transport in electrolytic process and the stereochemistry of hydrodimeric products were discussed.
\end{abstract}

\section{INTRODUCTION}

Electroreduction of cinnamic acid and its derivatives have been extensively studied from both synthetic ${ }^{1-7)}$ and mechanistic ${ }^{6.8 .9)}$ aspects. It is evident that the product-selectivity is strongly influenced by solvents and supporting electrolytes. Two waves were observed in polarography of methyl cinnamate (1) in aprotic solvents such as DMF and acetonitrile, while merged into one wave when proton donor was added ${ }^{9}$. In fact, we selectively obtained methyl hydrocinnamate (2) in electrolysis of 1 with a $\mathrm{Pb}$ cathode in aqueous methanol. On the other hand, the predominant product in extremely dry aprotic solvent was methyl 2,3-diphenyl-5-oxocyclopentanecarboxylate $(4)^{7)}$. Proton donor seemed necessary for the formation of dimethyl 3,4diphenyladipate (3; $d l$ and meso $)^{4.6 .7)}$.

Most of the above electroorganic syntheses

Department of Electronic Chemistry, Tokyo Institute of Technology, 4259 Nagatsuta, Midori-ku, Yokohama, 227 Key words: Electroreduction of methy 1 cinnamate, Rotating cylinder electrode, Mass transfer, Product- and stereoselectivities

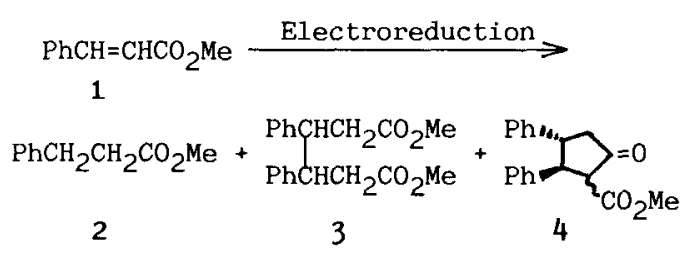

were carried out under controlled potential conditions. In this work, galvanostatic operations were performed for two reasons. One is that controlled current electrolysis is preferred in industry due to simplification of operation and installation. The other is the ease of elucidation of mass-transfer phenomena under controlled current conditions. As we have reported recently ${ }^{10-12)}$, the product-selectivity in galvanostatic electrolyses is strongly dependent on mass-transfer conditions. For example, hydrodimeric products are favored at higher mass-transfer rates in the electrohydrodimerization (EHD) of carbonyl compounds and acrylonitrile. Therefore, it is expected that the product-selectivity for the hydrodimers ( 3 and 4) can be controlled by controlling masstransfer rate in the electroreduction of cinnamic acid ester.

Furthermore, since any reaction pathway 
which determines the stereochemistry of the hydrodimers has not been completely clear yet, it is also expected to obtain new valuable informations from the influence of mass-transfer rate on the stereochemistry of $\mathbf{3}$ and $\mathbf{4}$.

A rotating cylinder electrode system has been applied in preparative-scale electrolytic syntheses ${ }^{13)}$ because of its substantially large surface area, combined with high mass transfer. In a recent study ${ }^{11)}$, we have showed it to be also a useful tool for elucidating the role of mass transport in the product-selectivity control. Hence, we purposively used a rotating cylinder electrode in this work.

\section{EXPERIMENTAL}

Electrolytic cell. The cathode was a $\mathrm{Cu}$ solid cylinder, $5 \mathrm{~cm}$ in length and $0.6 \mathrm{~cm}$ in diameter. A cylindrical ceramic diaphragm was used to separate catholyte from anolyte in a beaker type batch cell. The rotating cathode was driven by a variable speed motor (Yanagimoto RP-3).

Procedure. Before electrolysis, the cathode was polished with $1.0 \mu \mathrm{m} \mathrm{Al}_{2} \mathrm{O}_{3}$ (Marumoto Kogyo Co.) and rinsed twice with distilled water for $10 \mathrm{~min}$ in an ultrasonic bath, and then dried. The catholyte was a mixture of methanol and DMF with a volume ratio of $10: 90$, containing $A c O H, A c O N a$, methyl cinnamate, and $0.1 \mathrm{M} \mathrm{Bu}_{4} \mathrm{NBr}$. After electrolysis the catholyte was poured into an aqueous $\mathrm{AcONa} / \mathrm{ACOH}$ buffer solution, and repeatedly extracted with ether. The combined extract was washed with a saturated $\mathrm{NaCl}$ solution, and then dried over $\mathrm{Na}_{2} \mathrm{SO}_{4}$. After filtration, ether was evaporated and the residue was dissolved in acetonitrile. A definite amount of methyl benzoate was added to the sample solution as an internal standard for GC and HPLC analyses.

All the electrolyses were carried out at $27 \pm 2^{\circ} \mathrm{C}$.

Materials. Methyl cinnamate (1) was commercially supplied and recrystallized from ether/hexane. The compound 2 was prepared by the esterification of hydrocinnamic acid. The compounds 3 and 4 were isolated from the electrolytic solution by the recrystallization of the residue obtained above, using
$\mathrm{MeOH}$ as solvent. The melting point of the isolated 3 was $173-175^{\circ} \mathrm{C}$, indicating meso-3 (lit. ${ }^{14)}, \mathrm{mp} 175-176^{\circ} \mathrm{C}$ ). Although $d t-3$ was always produced more than meso- 3 , its isolation by recrystallization failed. The melting point of 4 was $126-127^{\circ} \mathrm{C}$ (lit. ${ }^{7}$, $\mathrm{mp} 126-$ $127^{\circ} \mathrm{C}$ ). In EHD of ethyl cinnamate, it was observed that only the trans-form of the ethyl ester analogue of 4 was obtained ${ }^{5.6)}$. Although the stereochemistry of 4 electrochemically formed was not reported, heating of 4 in $\mathrm{HBr} / \mathrm{EtOH}$ under reflux gave trans-3,4diphenylcyclopentanone (5), $\mathrm{mp} 175-176^{\circ} \mathrm{C}$ (lit. ${ }^{15)}, \mathrm{mp} 177^{\circ} \mathrm{C}$ ) as in the case of the ethyl ester analogue ${ }^{15}$ ). Hydrolysis of trans -4 , which is stereochemically equivalent to $d \tau-3$, yielded $a \imath-3,4$-diphenyladipic acid $(6)^{15}$. Heating of $a l-6$ in $10 \% \mathrm{H}_{2} \mathrm{SO}_{4} / \mathrm{MeOH}$ under reflux gave the desired $a l-3, \mathrm{mp} 72^{\circ} \mathrm{C}$ (lit. ${ }^{14}$ ), $\mathrm{mp} 73-74^{\circ} \mathrm{C}$ ).

Analysis. Quantitative analyses of the products were carried out on a Shimazu $6 \mathrm{~A}$ gas chromatograph (GC) using a $2 \mathrm{~m}$ column of $\mathrm{OV}-17$ on Chromsorb W (130 to $240^{\circ} \mathrm{C}$, $10^{\circ} \mathrm{C} \min ^{-1}$ ). However, because the GC retention time of $d l-3$ was equal to that of meso-3, their ratio was determined by the following method: The sum of their amounts was measured by GC and the amount of meso3 was measured by HPLC on a LiChrosorb Si 60 column (Merck) (Hexane : ethyl acetate $=7: 1$ ). Thus, subtraction of the amount of meso-3 from the sum afforded the amount of $d \imath-3$.

\section{RESULTS AND DISCUSSION \\ 3.1 Effect of addition of acetic acid}

Without adding acetic acid, material balance was usually poor. The main reason was that catholyte became basic during electrolysis and products were hydrolyzed to unknown compounds. Hence acetic acid (10-90 mM) and $\mathrm{AcONa}(5 \mathrm{mM})$ were added. The addition of acetic acid significantly influenced product distribution. As shown in Table 1, the current efficiency for 4 decreased from 55 to $0.78 \%$ as the initial concentration of acetic acid increased from 10 to $90 \mathrm{mM}$, while the current efficiency for 2 increased three times. On the other hand, the addition of acetic acid in- 
creased the ratio of 3 to 4 . However, the current efficiency for 3 showed a maximum at $50 \mathrm{mM}$ acetic acid, and decreased at higher acetic acid concentrations.

The relation between the current efficiency for 2 and the concentration of acetic acid can be explained from mechanistic aspect. A molecule of 1 moves from bulk solution to electrode surface and accepts one electron to form an anion radical (7), which is reduced only at more negative potential. The anion radical 7 abstracts one proton from proton donor to form a neutral radical (8), which is more easily reduced than 1 . This is why the two reduction waves observed in aprotic media merge into one wave as proton donor is added ${ }^{9}$.

$$
1 \stackrel{\mathrm{e}}{\longrightarrow}\left[\mathrm{PhCH}=\mathrm{CHCO}_{2} \mathrm{Me}\right]: \stackrel{\mathrm{H}^{+}}{\longrightarrow} \underset{7}{\longrightarrow} \underset{8}{\stackrel{\mathrm{CHCH}_{2} \mathrm{CO}_{2} \mathrm{Me}}{ }}
$$

Table 1. Influence of acetic acid concentration on electroreduction of methyl cinnamate (1). Catholyte: $10 \% \mathrm{MeOH} / \mathrm{DMF}, 80 \mathrm{mM}$ 1, AcOH, $5 \mathrm{mM} \mathrm{AcONa}$ and $0.1 \mathrm{M} \mathrm{Bu}_{4} \mathrm{NBr}$. Current density: $8 \mathrm{~mA} \mathrm{~cm}^{-2}$. Rotating speed: $250 \mathrm{rpm}$. Charge passed: $0.5 \mathrm{~F} \mathrm{~mol}^{-1}$.

\begin{tabular}{cccccccl}
\hline \multirow{2}{*}{ Run } & $\mathrm{AcOH} /$ & \multicolumn{3}{c}{ Current efficiency/\% } & $\mathbf{3 + 4}$ & \multirow{3}{*}{$\mathbf{3}$} \\
\cline { 3 - 5 } & $\mathbf{m M}$ & $\mathbf{3}$ & $\mathbf{4}$ & $\mathbf{2}$ & $\mathbf{4}$ \\
\hline 1 & 10 & 21 & 12 & 55 & 3.2 & 0.22 \\
2 & 30 & 22 & 31 & 32 & 2.9 & 0.97 \\
3 & 50 & 36 & 38 & 9.1 & 1.3 & 4.2 \\
4 & 70 & 58 & 16 & 3.4 & 0.33 & 4.7 \\
5 & 90 & 62 & 6.6 & 0.78 & 0.12 & 8.5 \\
\hline
\end{tabular}

\subsection{Stereochemistry of hydrodimers}

Stereochemistry of $\mathbf{3}$ and $\mathbf{4}$ found under various electrolytic conditons are shown in Table 2. The formation ratio of $d l$ - to meso-3 increased with an increase in the amount of acetic acid (Runs 1-3), while the trend was contrary as the concentration of 1 increased (Runs $3,6,7$ ). Considering that an increase in 1 concentration lowered the concentration ratio of acetic acid to 1 in the latter, the both phenomena were consistent with each other. On the other hand, the concentration of proton donor in the vicinity of cathode can be also controlled by current density and mass transfer. A high current density results in a high rate of proton consumption, leading to the lowering of the concentration of proton donor. On the contrary, the high concentration of proton donor is obtained by high masstransfer rate. Experimental results can be rationalized by the above described effects of current density and mass transfer (Runs 3 , 8-12).

Influences of electrolytic conditions on the ratio of $d l-3$ to trans -4 showed a tendency similar to those on the ratio of $a l-$ to meso-3 (Table 2). This means that trans $\mathbf{- 4}$ is favored when proton donor is deficient in the vicinity of the cathode. As described above, trans -4 is stereochemically equivalent to $d l-3$. Although either of both can be selectively yielded under proper electrolytic conditions, the ratio of the sum of $d l-3$ and trans -4 to meso-3 is almost constant $(5.3 \pm 0.1)$.

\subsection{Influences of current density and rotating speed}

Figure 1 shows the dependence of current efficiency for 3 on current density. There appear maxima of current efficiency for 3 at 12 . 20 and $30 \mathrm{~mA} \mathrm{~cm}^{-2}$, which are corresponding to the rotating speeds of 250,500 and 1000 rpm, respectively. This fact indicates that a higher mass-transfer rate gives a maximum current efficiency for 3 at a higher current

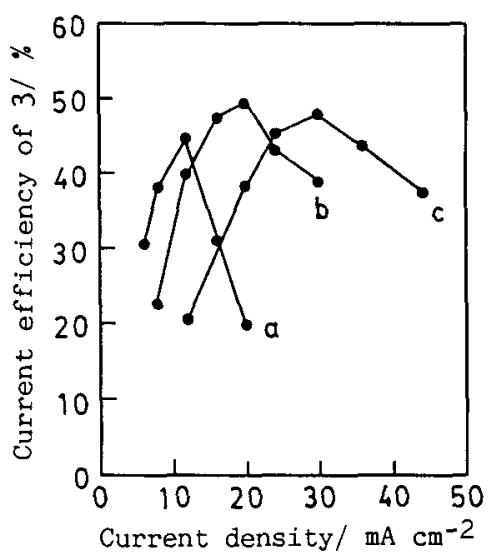

Fig. 1. Influence of current density on current efficiency for 3 at various rotating speeds: a, $250 \mathrm{rpm}$; b, $500 \mathrm{rpm}$; c. $1000 \mathrm{rpm}$. Catholyte: $10 \% \mathrm{MeOH} / \mathrm{DMF}, 80$ $\mathrm{mM}$ 1, $50 \mathrm{mM} \mathrm{AcOH}, 5 \mathrm{mM} \mathrm{AcONa}$ and $0.1 \mathrm{M} \mathrm{Bu}_{4} \mathrm{NBr}$. Charge passed: $0.5 \mathrm{~F} \mathrm{~mol}^{-1}$. 
Table 2. Stereochemistry of hydrodimeric products ( $d l-3$, meso-3 and trans-4) under various electrolytic conditions ( $\Omega$ : rotating speed; $i$ : current density). Catholyte: $10 \% \mathrm{MeOH} / \mathrm{DMF}, 1, \mathrm{AcOH}, 5$ $\mathrm{mM} \mathrm{AcONa}$ and $0.1 \mathrm{M} \mathrm{Bu}_{4} \mathrm{NBr}$. Charged passed: $0.5 \mathrm{~F} \mathrm{~mol}^{-1}$.

\begin{tabular}{|c|c|c|c|c|c|c|c|c|}
\hline Run & $1 / \mathrm{mM}$ & $\Omega / \mathrm{rpm}$ & & $\mathrm{cm}^{-2}$ & $\begin{array}{c}\mathrm{AcOH} / \\
\mathrm{mM}\end{array}$ & $\frac{d l-3}{m e s o-3}$ & $\frac{d l-3^{a)}}{\operatorname{trans}-4}$ & $\frac{d t-3+\operatorname{trans}-4}{\text { meso }-3}$ \\
\hline 1 & 80 & 250 & & 8 & 10 & 0.09 & $0.02(0.21)$ & 5.3 \\
\hline 2 & 80 & 250 & & 8 & 30 & 2.1 & $0.65(0.96)$ & 5.3 \\
\hline 3 & 80 & 250 & & 8 & 50 & 4.0 & $3.0(3.8)$ & 5.3 \\
\hline 6 & 120 & 250 & & 8 & 50 & 2.1 & $0.64(0.95)$ & 5.3 \\
\hline 7 & 160 & 250 & & 8 & 50 & 0.4 & $0.09(0.31)$ & 4.7 \\
\hline 8 & 80 & 250 & & 12 & 50 & 3.9 & $2.7(3.4)$ & 5.3 \\
\hline 9 & 80 & 250 & & 20 & 50 & 3.6 & $2.6(3.3)$ & 5.1 \\
\hline 10 & 80 & 500 & & 20 & 50 & 3.9 & $2.8(3.5)$ & 5.4 \\
\hline 11 & 80 & 1000 & & 20 & 50 & 4.0 & $3.1(3.8)$ & 5.3 \\
\hline
\end{tabular}

a) Values in parentheses are the ratios of the sum of $a l$-and meso-3 to trans-4.

density.

A similar phenomenon is also observed in Fig. 2, showing the dependence of current efficiency for $\mathbf{4}$ on current density. The current densities giving the maxima of current efficiencies for 4 at 250,500 and $1000 \mathrm{rpm}$ are equal to those in Fig. 1.

As illustrated in Fig. 3, the current density $\left(i_{\max }\right)$ giving the maximum current efficiencies for 3 and 4 falls on a straight line passing the origin. Here, $\Omega$ is the rotating speed of a cylinder electrode. The abscissa of Fig. 3

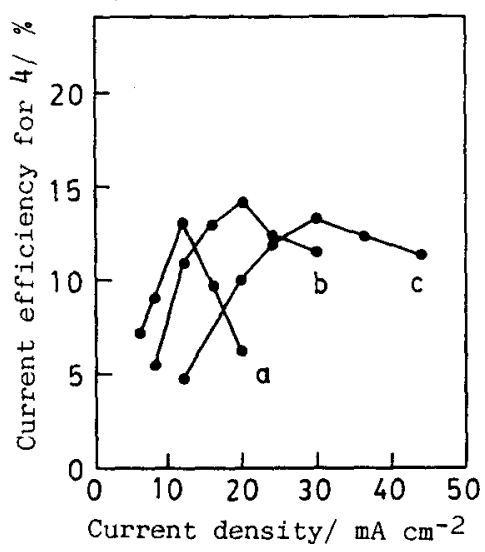

Fig. 2. Influence of current density on current efficiency for 4 at various rotating speeds: a, $250 \mathrm{rpm}$; b. $500 \mathrm{rpm}$ : c. $1000 \mathrm{rpm}$. Experimental conditions: given in Fig. 1. represents mass transfer coefficient $\left(k_{\mathrm{m}}\right)$ since $\Omega^{0.7}$ is proportional to $k_{\mathrm{m}}$ at a rotating cylinder electrode ${ }^{16,17)}$. The limiting current density for the formation of the intermediate 7 from 1 is also proportional to $k_{\mathrm{m}}$. Furthermore, a decrease in the total current efficiency $(2+3+4)$ was observed at current density exceeding $i_{\max }$. Hence the maximum phenomena in Figs. 1 and 2 are considered to be related to the mass transfer of 1 .

A low concentration of proton donor favors the formation of hydrodimers as shown in Table 1. Since an increase in current density leads to the consumption of more protons at unit time and decreases the concentration of proton donor at electrode surface, the

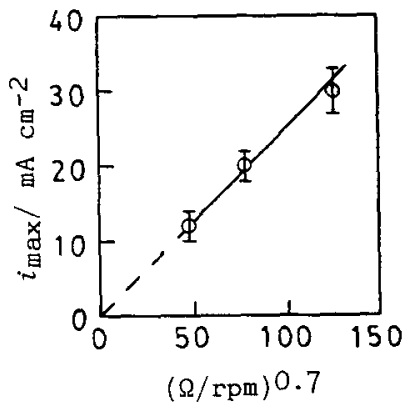

Fig. 3. Relation between $i_{\max }$ and $\Omega^{0.7}, i_{\text {max }}$ : $12 \pm 2,20 \pm 2$ and $30 \pm 3 \mathrm{~mA} \mathrm{~cm}^{-2}$ corresponding to 250,500 and $1000 \mathrm{rpm}$. respectively. 
phenomena observed in Figs. 1 and 2 when current density $(i)$ is less than $i_{\max }$ can be rationalized.

When $i<i_{\max }$, the current efficiency for hydrodimers at a lower rotating speed is higher than that at a higher rotating speed. This fact is explained similarly as above, because a decrease in rotating speed decreases the concentration of proton donor at the electrode surface.

It is interesting that the phenomena observed in EHD of 1 are evidently different from those of either carbonyl compounds or acrylonitrile.

Correlations of current density and rotating speed with current efficiency for 2 seem to be complicated. As shown in Fig. 4, at $250 \mathrm{rpm}$, current efficiency for 2 decreases with an increase in current density, reaches a minimum at $12 \mathrm{~mA} \mathrm{~cm}^{-2}$, and then increases at higher current density. On the other hand, an increase of current density at $1000 \mathrm{rpm}$ reduces monotonously the current efficiency. At $500 \mathrm{rpm}$, a transient behavior is exhibited: a small minimum followed by a decrease in current efficiency is found. This is considered to be due to interaction between the masstransfer processes of $\mathbf{1}$ and proton donors. However, a complicated solution system was

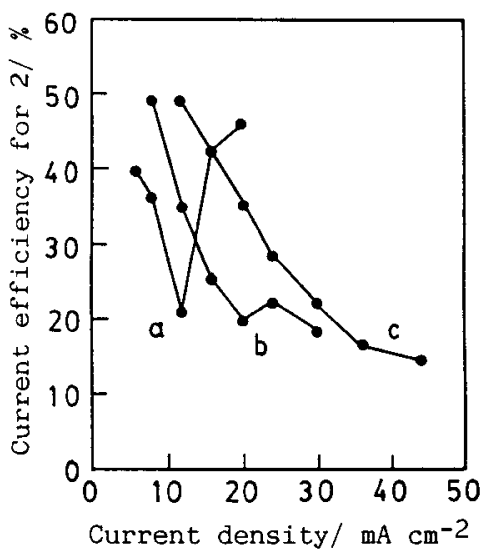

Fig. 4. Influence of current density on current efficiency for 2 at various rotating speeds: $a, 250 \mathrm{rpm} ; \mathrm{b}$, $500 \mathrm{rpm}: \mathrm{c}, 1000 \mathrm{rpm}$. Experimental conditions: given in Fig. 1.

used in this work to display significant product-selectivity control. Possible proton donors in the protonation of 7 and the hydrogen evolution are methanol, acetic acid and a trace of water. Therefore, their respective contributions to the formation of $\mathbf{2}$ must be also considered.

\subsection{Reaction mechanism}

Fig. 5. Reaction scheme of electroreduction of methyl cinnamate. 
In a previous study6), a reaction mechanism for EHD of cinnamic acid ester was proposed (Fig. 5). Although the most possible reaction pathway for EHD of acrylonitrile (AN) is oneelectron transfer to AN followed by nucleophilic addition of the resulting anion radical to unreacted $\mathrm{AN}^{9.12 .18)}$, this pathway is negated in EHD of cinnamic acid derivatives from an electroanalytical chemistry aspect ${ }^{8.9}$. Paths $A$ and $B$ in Fig. 5 were supposed to be prevailing in protolytic and aprotic media, respective$\left(y^{6)}\right.$. A fact that the formation ratio of ( $d z-$ $3+$ trans -4$) /$ meso- 3 is almost constant regardless of electrolytic conditions implies either that Path $A$ is negligible in this work or that the dl/meso ratios in the both paths must be nearly equal. However, the $d l / m e s o$ ratios in the protolytic and aprotic media had been found to be rather different ${ }^{6}$. Therefore, Path $A$ was hardly considered even though the catholyte contained a considerable amount of proton donor.

The reaction mechanism for the formation of 2 is somewhat confusing. Dianions derived from the direct two-electron transfer to 1 may also lead to the formation of 2 . However, the reduction pontential of $\mathbf{1}$ to the dianion is much more negative than that of hydrogen evolution. Therefore the dianion route (Path D) seems negligible. Consequently, a plausible reaction pathway (Path $\mathrm{C}$ ) is one-electron transfer to the neutral radical (8) followed by protonation.

A distinct limiting current for the oneelectron reduction of 1 appears on a voltammogram at $\mathrm{Hg}$ cathode ${ }^{9)}$. However, in this work the $\mathrm{Cu}$ cathode gave no limiting current, probably due to the overlapping of hydrogen evolution. Thus, it is difficult to clearly discuss the influence of cathode potential, even if it might be one of important factors.

\section{CONCLUSION}

In this work, two facts are clarified:

1. A high mass-transfer rate is necessary to obtain an optimum current efficiency for hydrodimers at a high current density.

2 . The formation ratio of $(d t-3+$ trans 4)/meso-3 is almost constant (ca. 5) regardless of electrolytic conditions. This suggests that Path $\mathrm{B}$ is predominant in an aprotic medium.

Although we also show that interaction between the mass-transfer processes of 1 and proton donors influences the productand stereo-selectivity, the correlation of these mass-trasfer processes has not been clear yet and further investigation is required.

\section{REFERENCES}

1) C. L. Wilson and K. B. Wilson, Trans. Electrochem. Soc., 84, 153 (1943).

2) S. Ono, Nippon Kagaku Zasshi, 76, 631 (1955).

3) M. M. Baizer and J. D. Anderson, J. Electrochem. Soc., 111, 223 (1964).

4) J. P. Petrovich, M. M. Baizer, and M. R. Ort, J. Electrochem. Soc., 116, 749 (1969).

5) L. H. Klemm and D. R. Olson, J. Org. Chem., 38, 3390 (1973).

6) H. Kanetsuna and T. Nonaka, Denki Kagaku, 49, 526 (1981).

7) I. Nishiguchi and T. Hirashima, Angew. Chem. Int, Ed. Engl., 22, 52 (1983).

8) V. J. Puglisi and A. J. Bard, J. Electrochem. Soc., 119, 829 (1972).

9) L. Lamy, L. Nadjo, and J. M. Savéant, J. Electroanal. Chem., 42, 189 (1973).

10) P. C. Cheng and T. Nonaka, J. Electroanal. Chem., 269, 223 (1989).

11) P.-C. Cheng, T. Nonaka, and T.-C. Chou, Bull. Chem. Soc. Jpn., 64, 1911 (1991).

12) P. -C. Cheng and T. Nonaka, Denki Kagaku, 60, 131 (1992).

13) H. V. K. Udupa, "Technique of Electroorganic Synthesis, Part 3. Scale up and Engineering Aspects", ed. by N. L. Weinberg and B. L. Tilak, John Wiley \& Sons, New York (1982), Chap. 8.

14) D. Y. Curtin and S. Dayagi, Can. J. Chem., 42, 867 (1964).

15) Z. L. Totton, R. C. Freeman, H. Powe11, and T. L. Yarboro, J. Org. Chem., 26, 343 (1961).

16) M. Eisenberg, C. W. Tobias, and C. R. Wilke, J. Electrochem. Soc., 101, 306 (1954).

17) D. R. Gabe and F. C. Walsh, J. Appl. Electrochem., 13, 3 (1983).

18) G. Piccardi, L. Nucci, F. Pergola, and R. Guidelli, J. Electroanal, Chem., 164, 145 (1984). 